\title{
Psychophysiological measures of anesthetic depth
}

\author{
SANDY VENNEMAN, A. MICHAEL ANCH, and JEFF DYCHE \\ Saint Louis University, St. Louis, Missouri
}

\begin{abstract}
The effects of isoflurane at three different doses $(0.75,1.68$, and $2.17 \mathrm{MAC})$ were examined in 12 male rats at two different times of day on baseline and in response to a tactile stimulus (tailpinch) and an auditory stimulus (click). Dependent measures included anterior-parietal and posterior-parietal electrocorticograms (ECoGs), heart rate (HR), respiration, and gross body movement. The ECoG was the measure most sensitive in distinguishing anesthetic dose, whereas gross body movement exhibited the least sensitivity. Finally, although there were significant circadian variations in the ECoG, HR, and respiration for baseline doses of isoflurane, circadian variation in anesthetic action was not demonstrated. The data support the notion that animals can experience stimuli under anesthesia to which they do not, or cannot, exhibit motor responses.
\end{abstract}

Veterinarians and physicians use observation of gross muscle movement, heart rate, and respiration as indicators of anesthetic depth and pain perception during anesthesia. These common methods may not offer enough sensitivity to avoid errors. This is demonstrated in anecdotal accounts of patients describing painful sensations during incision and subsequent surgery without being able to communicate their pain (Shapiro, 1996). Electrocorticogram (ECoG)/electroencephalogram (EEG) in human monitoring may offer a better alternative for sensitive monitoring of pain perception. ECoG/EEG shows changes dependent upon auditory stimulation. If changes due to auditory stimulation mimic changes produced by tactile stimulation, perception of pain would be difficult to assess with ECoG/EEG, because of naturally occuring noise in the operating room. To be able to discriminate, we employed auditory stimulation as a variable in this investigation.

The following questions were addressed in this study: "Do measures of stimuli perception indicate pain is perceived when movement does not occur?" "Are the traditional indicators used in the operating room such as respiration, heart rate, and gross muscle movement as sensitive as ECoG/EEG for monitoring pain perception?" "Does reaction to stimulation differ between auditory and tactile sensory modalities as a function of anesthetic depth?" "Are there differences due to the time of day a procedure is performed?" The answers to these questions may have applicability to reports of humans who perceive, remember and, under some circumstances, learn new associations while under general anesthesia.

Anesthetic depth in relation to stimulus detection and circadian rhythms in various modalities has received only

Correspondence should be addressed to $S$. Venneman, University of California, Los Angeles Neuropsychiatric Institute, 760 Westwood Plaza, Room 37-441, Los Angeles, CA 90024 (e-mail: sandy@qeeg. ucla.edu). moderate attention. Historically, various traditional measures (e.g., respiration, heart rate, tactile stimulation, etc.) have been used to determine depth of anesthesia (Barraco, Phillis, Campbell, Marcantonio, \& Salah, 1989; Loper, Reitan, Bennet, Benthuysen, \& Snook, 1987; Merkel \& Eger, 1963).

Other less established benchmarks of anesthesia depth, such as EEG, have been used sparsely (Merkel \& Eger, 1963; Nayak, Roy, \& Sharma, 1994; Reddy, Moorthy, Mattice, Dierdorf, \& Deitch, 1992; Thomsen, Christensen, \& Rosenfalck, 1989). However, these analyses show consistent trends reflecting anesthetic depth (Clark et al., 1973; Dripps, Eckenhoff, \& Vandam, 1977; Eger, Stevens, \& Cromwell, 1971; Rampil et al., 1988; Schwilden \& Stoeckel, 1987; Yli-Hankala, Eskola, \& Kaukinen, 1989). Most notable in these assessments is burst suppression, the inhibition of high amplitude EEG activity. This indicates decreased cerebral metabolism, and it is noted as a signature of depth in anesthesia (Engelhardt, Carl, Dierks, \& Maurer, 1991; James, Volgyesi, \& Burrows, 1986; Pauca \& Dripps, 1973; Rampil et al., 1988; Schwilden \& Stoeckel, 1987).

Isoflurane (1-chloro-2,2,2-trifluroethyl-difluromethyl ether), an inhalation anesthetic, has become one of the most widely used anesthetics in the United States during the past 15 years. Its physical properties and actions on various organ systems are well documented (e.g., Eger, 1985). In spite of this, its ECoG activity has not been widely studied (Schwilden \& Stoeckel, 1987; Thomsen et al., 1989), and circadian influences remain unknown. Since circadian rhythms exert influence with other anesthetics, this is a topic that needs to be addressed (Munson, Martucci, \& Smith, 1970; Reinberg \& Halberg, 1971).

We attempted to investigate the ability of rats to detect sensory stimuli under three doses of isoflurane during two extremes of their circadian cycle. To do this, we compared two sensory modalities, tactile (tailpinch) and auditory (clicks) stimulation, in order to examine differences in responses to painful and nonpainful stimuli and 


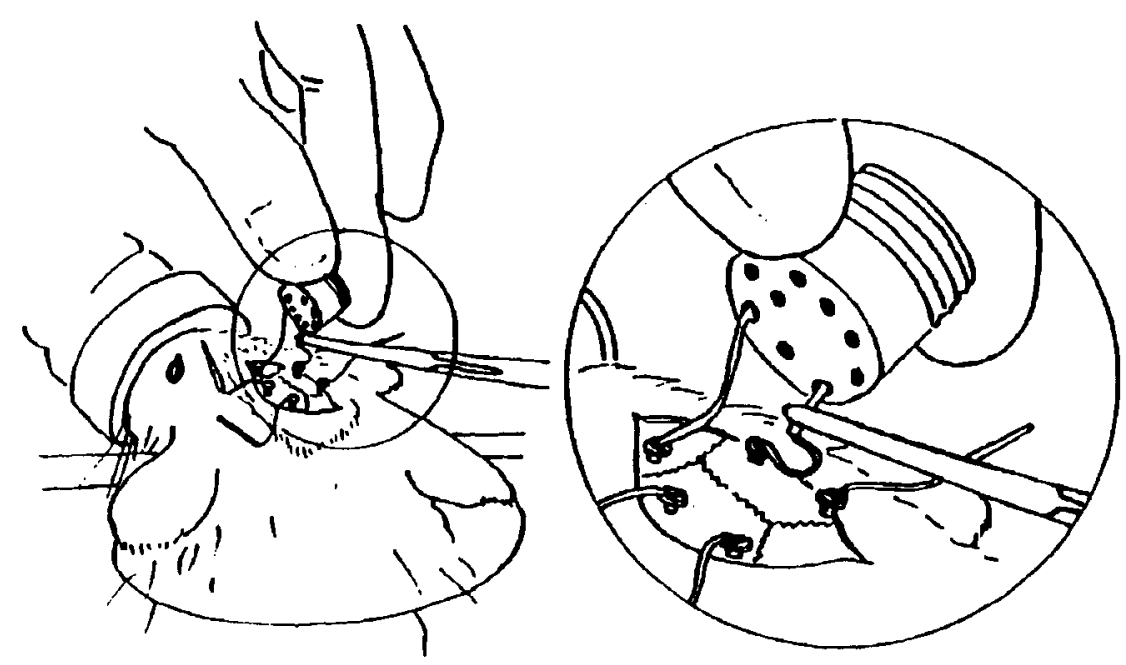

Figure 1. The electrode array consisted of a ground plus bipolar recording from homologous sites in two different regions of the cortex: anterior parietal and posterior parietal regions of the cortex.

to examine possible partitioning of anesthetic depth as a function of sensory modality.

\section{METHOD}

\section{Subjects}

Twelve male Sprague-Dawley rats weighing between 279 and $380 \mathrm{~g}$ were used. Only mature rats, $4-5$ months of age, were used, in order to avoid variations in anesthetic action seen in the very young or old (Eger, 1985; Eger et al., 1971; Gregory, Eger, \& Munson 1969; Stevens et al., 1975). The rats were housed individually in a climate-controlled atmosphere and maintained on a 12:12-h light:dark cycle. The animals were fed a standard diet of rat chow and ad-lib water and were handled daily during a 30-day acclimation period.

\section{Procedure}

Isoflurane was used as the sole anesthetic agent for all procedures, including preexperimental implantation, to avoid possible effects of drug interaction. The rats were implanted with stainless steel screw electrodes for ECoG recordings according to standard procedures (Bergman, Winter, Rosenberg, \& Reschtaffen, 1987). The electrode array consisted of a ground plus bipolar recording from homologous sites in two different regions of the cortex: anterior parietal (AP) and posterior parietal (PP) regions of the cortex (Figure 1). Different regions were examined since there is some evidence of ECoG variation dependent on region (Clark \& Rosner, 1973; Tinker, Sharbrough, \& Michenfelder, 1977).

Following implantation, a period of 7 days was permitted for adequate recovery and drug washout (Bahlman, Eger, \& Cromwell, 1972; Cromwell, Eger, Stevens, \& Dolan, 1971; Davison, Steinhelber, Eger, \& Stevens, 1975). On Day 8, isoflurane anesthesia was induced at $2.2 \%-3.5 \%$ in oxygen. Two electrodes $(1 \mathrm{~cm}$ in diameter) were secured to the skin, covering the cutaneous maximus muscle, to record heart rate (HR).

An ABS socket connected to a polygraph (Grass Model 78D) was attached to the surgically implanted ABS plug before the rat was transferred to the experimental anesthesia chamber (a Plexiglas cylinder with interior dimensions of $23 \times 14 \times 12 \mathrm{~cm}$ ). The rat was positioned to permit unobstructed breathing, exits were filled with synthetic clay, and a vaporizer was attached to the cham- ber. The isoflurane/oxygen mixture entered the chamber through a $0.5-\mathrm{cm}$ port at the rat's head and exited via a $0.6-\mathrm{cm}$ port toward the rat's posterior.

Following a 30-min equilibration, baseline measurements were taken. After this baseline period, experimental manipulations were performed. The baseline for the auditory stimulus was the halfepoch preceding the click (an epoch $=20 \mathrm{sec}$ ). For auditory stimulation, the results were compared with the baseline values immediately preceding each stimulus in order to control for residual effects of the preceding stimulus. The baseline for the tactile stimulus was a 12-epoch period (4 min), divided equally between the recordings at the very beginning of the new dose level to be studied and at the very end of the trial of that concentration. The results for tactile stimulation were compared with these baseline values.

Figure 2 illustrates the experimental design. The tactile stimulus consisted of a tailpinch of three-epoch (1-min) duration, during which observations of gross muscle movements and other measures (described below) were recorded according to standard procedures (Eger, Saidman, \& Brandstater, 1965; Merkel \& Eger, 1963; White, Johnston, \& Eger, 1974). Pressure of $9.0 \pm .02 \mathrm{~N}$ was applied to the distal third of the tail by an alligator clip (wrapped in tape to reduce tissue damage). We placed the clip on the portion of the tail that measured $7 \mathrm{~mm}$ in diameter, ensuring consistent pressure across subjects.

One minute after the clamp was removed, a series of four auditory stimuli (single clicks) was presented at one epoch (20-sec intervals). The clicks were produced by a mechanical metal device at the level of the rat's head at a distance of $7-12 \mathrm{~cm}$. A biological comparison of the stimuli was performed employing a calibrated auditory brainstem response (ABR) unit. ${ }^{1}$ Each auditory stimulus was assessed to have an output in excess of $125 \mathrm{~dB}$ SPL. The tailpinch always preceded the auditory stimulus to control for possible conditioning effects (i.e., to prevent the auditory stimulus from serving as a discriminant stimulus for tactile stimulation). Such a conditioned response could result in self-induced analgesia from release of beta endorphins and enkephalins in anticipation of pain, as noted by other researchers (Bolles, 1982; Fanselow 1981; Meadows, Phillips, \& Davey, 1988). Following these stimuli, unstimulated 2-min baseline recordings were made.

Each experiment was conducted at each of the following three doses of mean alveolar concentration (MAC), traditionally defined as the concentration at which $50 \%$ of the subjects respond to aver- 


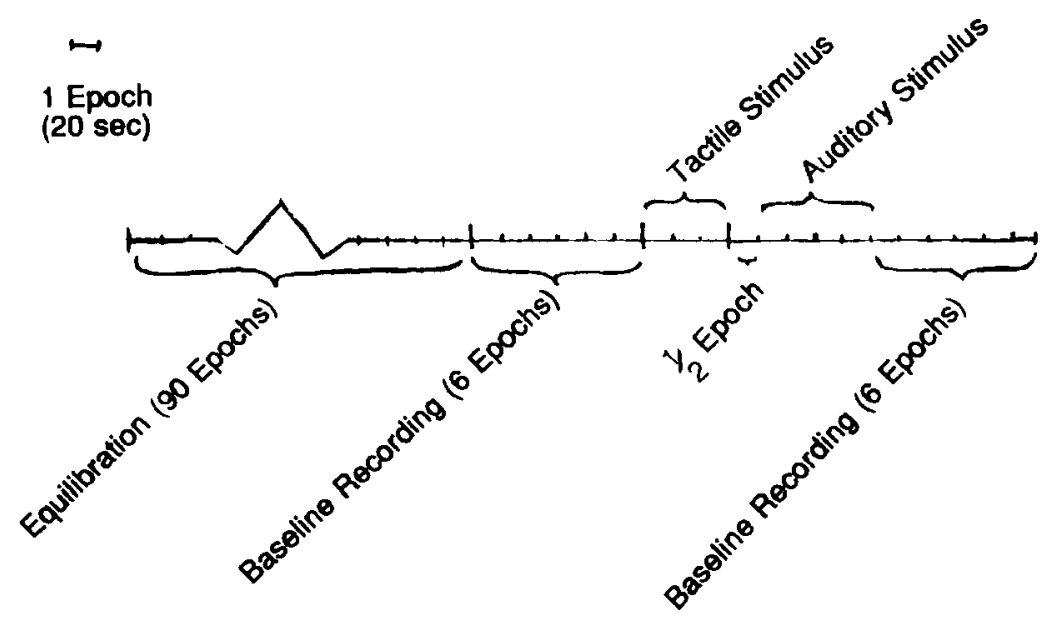

\begin{abstract}
Figure 2. The experimental procedure at one dose level at one time of day. This same procedure was performed for each of the three dose levels in the a.m. and again in the p.m., utilizing a balanced design.
\end{abstract}

sive stimulation (Dripps et al., 1977; Eger et al., 1965): 0.75 MAC, 1.68 MAC, and 2.17 MAC, as determined by calibrated values on a headbrink Kinet-O Meter vaporizer. The vaporizer was calibrated using a mass-spectrometer at the beginning and end of the study. Results were averaged, yielding the reported MAC values $(0.75 \pm$ $.03,1.68 \pm .05,2.17 \pm .07)$. Although it was our intent to have the anesthetic concentrations spread out to encompass 1 and $2.5 \mathrm{MAC}$ (and a midpoint), the exact values were determined by calibrating the vaporizer before and after the study with a mass-spectrometer and taking an average value for MAC. These concentration doses were chosen on the basis of pilot studies and on the basis of ethical standards, as well as limitations due to respiratory and blood pressure depressant characteristics of isoflurane. ${ }^{2}$ In addition, these concentration values were chosen in an attempt to reflect conditions found in surgery and in other studies (cf. Schwilden \& Stoeckel, 1987; Yli-Hankala et al., 1989).

Thirty minutes of equilibration was allowed after the vaporizer setting was adjusted to the next experimental concentration (e.g., Thomsen et al., 1989). An identical procedure was employed for all concentrations. Upon completion of trials, the rat was allowed a 7-day washout period.

Following this washout period, a second, identical experiment was conducted at the other circadian extreme (i.e., $180^{\circ}$ out of phase with regard to the time of day of the first experiment). If the first trial was in the a.m., the second trial was in the p.m., and vice versa. The a.m. and p.m. time periods ( $7 \mathrm{a} . \mathrm{m}$. and $8 \mathrm{p} . \mathrm{m}$.) refer to the light and dark periods, respectively. Initial dose of isoflurane and order of time of day were randomly assigned, in a manner to ensure that all possible orders of administration were balanced. A balanced design ensured that each order or dose administration was represented (i.e., $0.75,1.68,2.17 ; 1.68,2.17,0.75 ; 2.17,0.75,1.68$, etc., for a.m. and p.m.) so that any effects of order of presentation would be eliminated in the statistical analysis. This procedure resulted in each rat being examined at each of the three doses of the anesthetic twice: once in its most active portion of the daily cycle, and once in its least active portion. These trials were 1 week apart to ensure adequate drug washout.

\section{Data Analysis}

The use of visual evaluation, rather than spectral analysis, more closely parallels clinical application in the setting of an operating room. Therefore, electrophysiological recordings were scored by hand using 20-sec epochs. ECoG epochs were scored in terms of the number of waves exceeding $75 \mu \mathrm{V} /$ epoch unit; HR was scored in terms of beats/epoch. An ECoG amplitude of $75 \mu \mathrm{V}$ was chosen because waves of this magnitude indicate wakefulness in the rat (McGinty, 1967). Both respiration (scored as breaths per minute) and movement (recorded categorically as present or absent) were visually recorded, mirroring a clinical setting.

Repeated violation of sphericity dictated the use of a conservative multivariate approach to evaluate all data, with the exception of movement. To examine circadian variations independent of manipulation and to establish baseline rates, a series of multivariate analyses of variance (MANOVAs) was performed using the independent variables of time of day, stimulation type, and concentration of anesthetic using baseline data. These statistical analyses were assessed by examining the five dependent measures: HR, AP ECoG, PP ECoG, respiration, and movement (post hoc $t$ tests followed any significance). To assess the influence of manipulation under the anesthetic, additional analyses were performed using changes in $\mathrm{HR}, \mathrm{AP} / \mathrm{PP}$ ECoGs, and movement upon stimulation, relative to baseline.

Gross muscle movement was visually observed, as it would be in a clinical setting. It was scored categorically and was analyzed using Cochran's $Q$ with Scheffé-like post hoc tests performed on significant main effects. The McNemar nonparametric test was employed to analyze movement for time of day.

In summary, the design was, therefore, a repeated measures, balanced design, with three factors: concentration dose $(0.75 \mathrm{MAC}$, 1.65 MAC, and 2.17 MAC), stimulus type (tailpinch vs. click), and time of day ( 7 a.m. vs. 8 p.m.). During baseline (no stimulus presented), the effects of these three doses of MAC on HR, AP ECoG, PP ECoG, respiratory rate, and movement were assessed during the a.m. and p.m. conditions. Following baseline, the effects of the three factors (concentration, stimulus type, and time of day) and their interactions on the same physiological measures (HR, AP ECoG, PP ECoG, respiratory rate, and movement) were assessed. Change scores, rather than the absolute values of our dependent measures, were used in these statistical analyses to control for changes in the overall scores due to baseline shifts.

\section{RESULTS}

The data were analyzed in terms of baseline and stimulation conditions. For the tactile condition, baseline was defined as the initial six epochs ( $2 \mathrm{~min})$ plus the final 
Table 1

$F$ Statistics of Baseline and Stimulation Values

\begin{tabular}{|c|c|c|c|c|c|c|c|c|c|c|c|}
\hline \multirow[b]{2}{*}{ Variable } & \multicolumn{2}{|c|}{ Heart Rate } & \multicolumn{2}{|c|}{ AP ECoG } & \multicolumn{2}{|c|}{ PP ECoG } & \multicolumn{2}{|c|}{ Respiration } & \multicolumn{2}{|c|}{ Movement } & \multirow[b]{2}{*}{$d f$} \\
\hline & Baseline & Stimulation & Baseline & Stimulation & Baseline & Stimulation & Baseline & Stimulation & Baseline & Stimulation & \\
\hline Time & $35.32 \dagger$ & 0 & 3.11 & 0.52 & $6.47^{*}$ & 1 & $6.78^{*}$ & n.a. & & & $\overline{1,11}$ \\
\hline Dose & $13.594+t$ & $0.147_{t}^{+}$ & $69.969+$ & $12.454 t_{t}^{+}$ & $67.5134_{+}^{+}$ & $14.144 t_{+}^{+}$ & $31.255 \dagger_{+}^{+}$ & n.a. & & $Q \dagger$ & 2,10 \\
\hline Stimulus type & $7.16^{*}$ & $28.59 \dagger$ & $24.98 \dagger$ & 0.4 & $5.96^{*}$ & 4.42 & n.a. & n.a. & & $Q^{+}$ & 1,11 \\
\hline Time $\times$ dose & $3.445_{\ddagger}^{+}$ & $0.948_{+}^{+}$ & 2.198 & $2.95^{*+}$ & $2.275_{t}^{\ddagger}$ & $2.533_{+}^{+}$ & $1.31_{+}^{+}$ & n.a. & & & 2,10 \\
\hline Time $\times$ stimulus & 0 & 0.5 & 1.63 & $5.98^{*}$ & 3.94 & $11.511^{\circ}$ & n.a. & n.a. & & & 1,11 \\
\hline Dose $\times$ stimulus & $9.158+t$ & $0.429+$ & $9.166^{\dagger}$ & $7.378+t$ & $4.616^{*} \div$ & $5.831^{*}+$ & n.a. & n.a. & & & 2,10 \\
\hline Time $\times$ dose $\times$ stimulus & $3.609+$ & $0.313 \ddagger$ & $5.648^{*}$ & $1.862_{+}^{+}$ & $2.355 t$ & $3.986 * \ddagger$ & n.a. & n.a. & & & 2,10 \\
\hline
\end{tabular}

Note- $Q$, nonparametric tests performed on dichotomous variable (see text). ${ }^{*} p<.05 . \quad \dagger p<.01 . \quad \ddagger$ Multivariate $F$.

six epochs of each set. We averaged these two time periods to control for carry-over effects resulting from sensory stimulation, which resulted in more conservative (rather than liberal) results. The baseline and stimulation values measured were AP and PP lobe ECoG, HR, movement, and respiration. Table 1 summarizes all statistical results.

\section{Baseline Values}

An examination of baseline data was conducted for use as a reference in examining the action of the anesthetic using change scores. There was a significant main effect for the dose of anesthetic for all the variables measured, with the exception of movement. No movement occurred during any of the baseline recordings, as expected due to the fact that no stimuli were presented during baseline. All other variables (HR, AP and PP ECoG, and respiration) showed differences dependent upon the dose of the anesthetic. Even without statistical analysis, the difference between doses was readily apparent by mere visual examination of the ECoG record. This was particularly true of detection of $0.75 \mathrm{MAC}$ versus the two higher concentrations.
There was a highly significant effect for isoflurane concentration with AP ECoG $[F(2,10)=69.97, p<$ $.01]$ and PP ECoG $[F(2,10)=67.51, p<.01]$. Closer examination revealed that the statistically significant difference was between $0.75 \mathrm{MAC}$ and the other two concentrations. This is what was visually observed on the record. Figure 3 is an example of the ECoG recordings during baseline for the three doses of MAC used in this experiment. The sudden appearance of high-amplitude waves during periods of low-amplitude activity (bursting), which has been frequently reported, decreases as the depth of anesthesia increases. The difference in frequency of bursts between 1.68 and 2.17 MAC was not great enough during baseline recordings to be statistically significant.

Post hoc $t$ tests for paired samples showed a significant difference between a.m. and p.m. for $0.75 \mathrm{MAC}$ tailpinch $[t(11)=-3.65, p<.01]$. The significant three-way interaction for AP ECoG further demonstrates that the carry-over effect of the $0.75 \mathrm{MAC}$ concentration was great enough to be significant only in the a.m. Although there was no three-way interaction for PP ECoG,
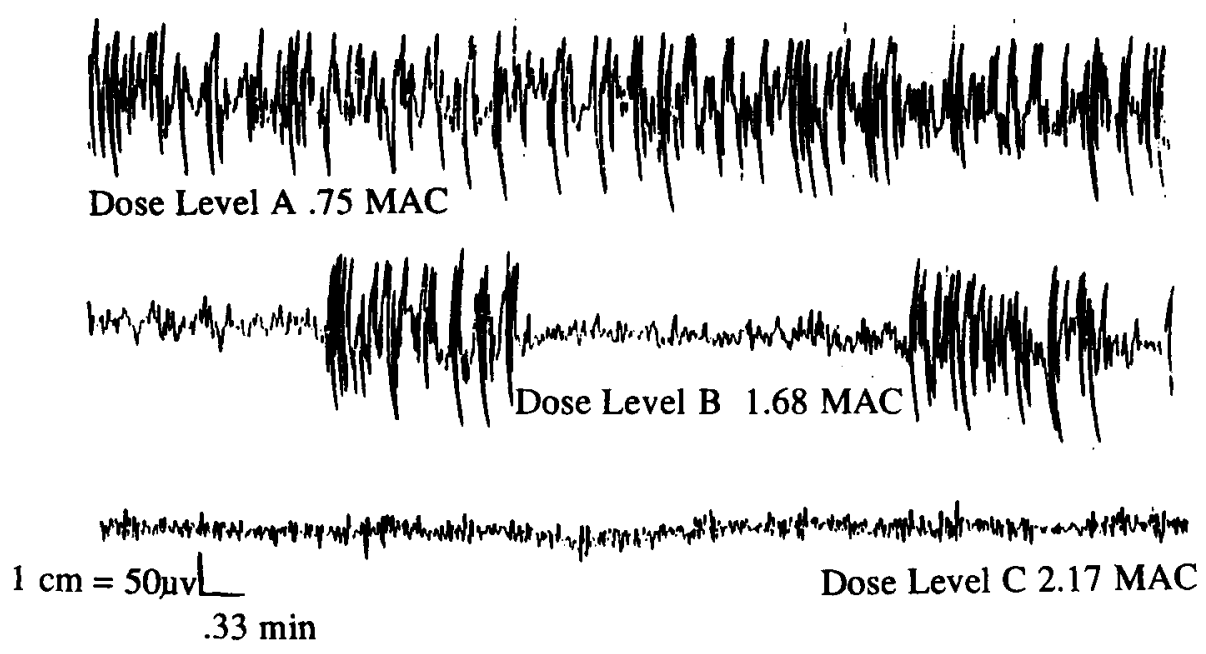

Figure 3. An example of the ECoG recordings during baseline for the three doses of MAC used in this experiment. High-amplitude waves during periods of low-amplitude activity (bursting) can be seen to decrease as the depth of anesthesia increases. The difference frequency of bursts between 1.68 and 2.17 MAC was not great enough during baseline recordings to be statistically significant. 


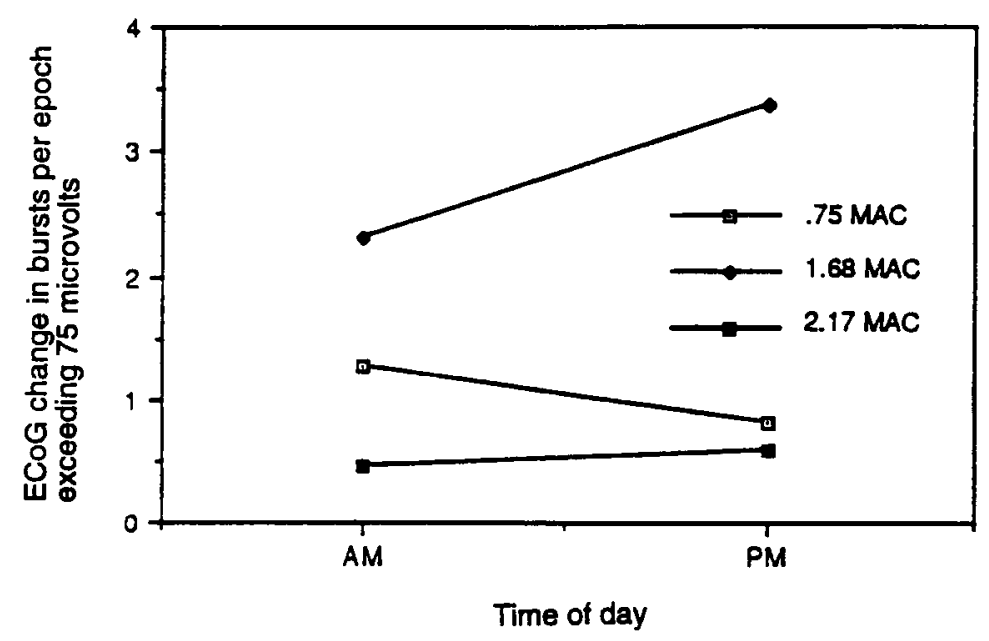

Figure 4. Change in AP ECoG demonstrating a significant interaction between time of day and dose of isoflurane. This two-way interaction illustrates the action of the anesthetic as a function of dose and time of day.

there was a main effect for time of day. Again, the 0.75 MAC dose level differed from the other two.

An interaction was found (dose $\times$ stimulus) for both AP and PP ECoG and HR baseline measures. In addition, two main effects were found for baseline respiration conditions. As anticipated, respiration was significantly higher in the p.m. condition (the rat's natural active period) $[F(1,11)=6.78, p<.05]$ than in the a.m. condition. Mean respiration rate (breaths/minute) for p.m. $=39.56$, and for a.m. $=36.72$. Mean breaths/ minute for all three doses were significantly different $[F(2,10)=31.26, p<.01]$. Mean breaths/minute for $0.75,1.68$, and $2.17 \mathrm{MAC}$ were $56.83,31.92$, and 25.67 , respectively.

From this baseline data, we can see that, by observing the undisturbed rat, we can accurately predict which dose of anesthetic it was administered. This was not, however, the purpose of the study. We wished to examine the action of the anesthetic by stimulating the animal under all three doses, and, now that we had a baseline to use as a comparison, this could be accomplished.

\section{Stimulation Values}

AP and PP ECoG. Table 1 summarizes the results of statistical analyses for AP and PP ECoG values following sensory stimulation. The results were similar. There was a highly significant dose-response effect for isoflurane of AP ECoG $[F(2,10)=14.14, p<.01]$ and PP ECoG $[F(2,10)=12.5, p<.01]$. They both showed a significant interaction between time of day and stimulus presentation [for AP ECoG, $F(1,11)=5.98, p<.05$; for PP ECoG, $F(2,10)=11.51, p<.01]$ and a significant interaction between the dose of isoflurane and stimulus presentation [for AP ECoG, $F(2,10)=7.4, p<.01$; for PP ECoG, $F(2,10)=5.83, p<.05]$.

There were also some differences in the statistical analyses of AP versus PP ECoG. Whereas there was a significant interaction between time of day and dose of isoflurane for AP ECoG $[F(2,10)=2.96, p<.05]$ (Figure 4), this did not quite reach significance for PP ECoG. Also, only PP ECoG showed a significant three-way interaction $[F(2,10)=3.99, p<.05]$ (Figure 5). A post hoc test revealed that this significance resulted from a circadian difference in reaction to tactile stimulation under $0.75 \mathrm{MAC}$ dose $[t(11), p<.01]$.

Heart rate. HR showed a significant effect for stimulus type between $0.75 \mathrm{MAC}$ and the other two doses $[F(1,11)=28.59, p<.01]$. Mean tactile change was 1.18 beats/epoch, whereas auditory change was -0.38 beats/epoch. The difference in algebraic sign expresses the fact that HR increased under conditions of tactile stimulation and decreased under conditions of auditory stimulation.

Movement. Movement also exhibited a main effect for dose and stimulus type: [Cochran's $Q(2)=10.00, p<$ .01]. A post hoc Scheffé-like comparison showed the only significant difference to be between the $0.75 \mathrm{MAC}$ concentration and the other two concentrations. No significant difference was found examining time of day employing the McNemar nonparametric test. Movement occurred only under the condition of tactile stimulation.

Respiration. No measure of respiration was possible during the stimulation due to its brevity.

\section{DISCUSSION}

This study reveals several findings that impact an analysis of anesthetic depth with isoflurane anesthesia. First, the ability to detect stimuli can occur in the absence of movement, as indicated by changes in the ECoG and HR independent of movement. This supports the notion that the brain can respond to sensory stimulation in the absence of behavioral responses to stimulation under anesthesia. From a clinical perspective, this is an experimental validation of anecdotal evidence that patients sometimes experience pain without the ability to perform gross muscle 


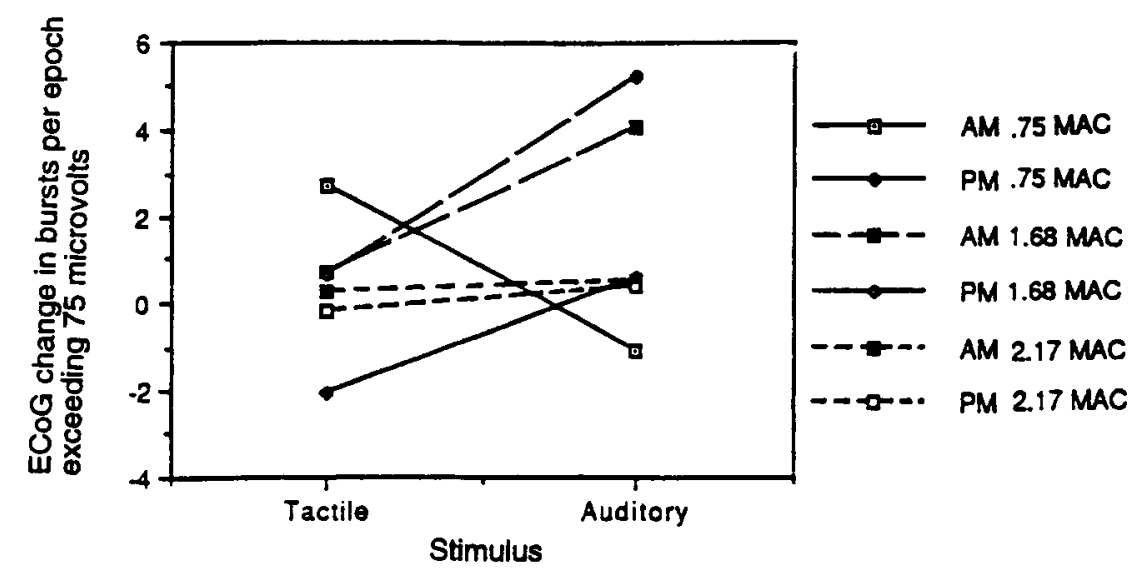

Figure 5. Change in PP ECoG demonstrating a significant three-way interaction. This three-way interaction illustrates the action of anesthetic as a function not only of dose level and time of day but also of stimulus type.

movements or vocalizations to express their plight. This would suggest that visual observation of gross motor movement is inadequate as a sensitive indicator of pain perception in clinical settings. Additionally, ECoG appeared to be a more sensitive indicator of anesthetic depth than HR. Second, stimulus type effects were differentiated, with auditory stimulus detection persevering longer (more refractory to anesthetic depth) than tactile stimulus detection. Thus, the results of this study, using isoflurane anesthesia, would support evidence that humans may continue to perceive information while under anesthesia. Lastly, circadian differences are detectable in isoflurane anesthesia; however, they do not affect responses to sensory stimulation. Each of these findings will be elaborated further.

\section{Stimulus Detection}

A critical feature in this experiment was the use of multiple dependent measures in assessing the anesthetic action of isoflurane. Such measures allow for a comparison of physiological systems, assessing possible synergistic effects and possible dissociation effects among the dependent measures.

Although isoflurane had profound effects on all dependent measures employed in this study (ECoG, HR, movement, and respiration), during baseline, the ECoG was the most sensitive to sensory stimulation. The stimulation values, not the baseline values, express the action of the anesthetic. Action determines whether or not stimuli are detected. In this study, gross muscle movement was not a statistically significant indicator of anesthetic depth or action, which supports the notion that this is a crude tool to determine whether stimuli are being detected. This lends credence to personal testimonies of pain perception during anesthesia. The ECoG data, on the other hand, demonstrated better sensitivity, as reflected in its ability to detect a difference in the action of isoflurane as a function of dose of anesthesia, stimulus type, and time of day. Indeed, many of these changes were visually apparent in the ECoG record itself. This would suggest that it would be advantageous to employ this measure in a clinical setring.

The ECoG tracings showed a very consistent and different pattern on the ECoG records for each of the three different doses of anesthetic, similar to shifts noted by others (Thomsen et al., 1989) and described as "burst suppression" (Pauca \& Dripps, 1973; Rampil et al., 1988; Schwilden \& Stoeckel, 1987). Not surprisingly, there was a definite affect of isoflurane dose on ECoGs and movement, compatible with increasing depth of anesthesia. In agreement with previous research, there was a dose-related burst suppression in the ECoG, along with an absence of movement at the two highest levels of isoflurane concentration.

These results are consistent with previous research on anesthetic depth and EEG recordings (Clark \& Rosner, 1973; Dripps et al., 1977; Pauca \& Dripps, 1973; Nayak et al., 1994; Schilden \& Stoeckel, 1987), manifesting burst suppression with increasing depth of anesthesia and an inverse relationship between anesthesia depth and ECoG amplitude. However, we were surprised to find that, in contrast to previous studies with other inhalation anesthetics (e.g., Halothane), there were no significant differences involving circadian variation in anesthetic action during manipulations.

\section{Circadian Effects}

Circadian influences on analysis of anesthetic depth are also noteworthy, since $\mathrm{ECoG}$ data indicate that time of day interacts with both isoflurane dose and stimulus type. In addition, it is well known that anesthetic agents show rhythms of effectiveness and toxicity (Nair \& Casper, 1969; Reinberg \& Halberg, 1971; Roberts, Turnbull, \& Winterburn, 1970).

In this study, however, we did not find a potent circadian effect for the action of isoflurane, as has been reported for other anesthetics (Emlen \& Kem, 1963; Pauley \& Scheving, 1964). The lack of a profound circadian ef- 
fect during sensory stimulation may have resulted from the small sample size $(N=12)$. This limited chances of finding significant effects. In addition, since multiple $t$ tests were used, an alpha of .01 was chosen for the post hoc analysis to control for Type I error. This further limited the possibility of statistical differences, but it increased confidence in the present findings.

\section{Stimulus Type}

There was a striking difference in the reaction to auditory versus tactile stimulation, as indicated by $\mathrm{HR}$ and ECoG values. ECoG responses occurred significantly more with auditory stimulation than with tailpinch. The animals never moved during the auditory stimulation, as they did during the painful stimulus. The results suggest that auditory stimulus detection is more resistant to sedating effects than is tactile stimulus detection, and they provide support for a modality-specific effect of anesthesia on arousal level. This is consistent with previous literature that has documented persistent hearing under anesthesia (Cheek, 1962, 1964; Pearson, 1961).

\section{ECoG}

Although ECoG recordings were from different sites (AP vs. PP sites), they exhibited similarities. Interestingly, both sites demonstrated a difference at $1.68 \mathrm{MAC}$ under the stimulation condition that was not present during baseline. Data for the baseline recordings did not show a significant difference between 1.68 and 2.17 MAC. This seemed to indicate that the two doses were not discrete. With auditory (but not tactile) stimulation, however, responses of the animal were significantly different for these two doses. This highlights the fact that depth of anesthesia seems to be information specific, both in terms of presence or absence of a sensory stimulus, as well as the sensory modality being stimulated. Thus, the results of the present study would support Merkel and Eger (1963), who failed to find a single physiological parameter that would be adequate as a sole measure of depth of anesthesia. This would have direct relevance to surgical considerations for both veterinary and human populations.

\section{Heart Rate}

The results showed that $\mathrm{HR}$ can indicate time of day (significantly higher rate at $0.75 \mathrm{MAC}$ than at 1.68 or 2.17 MAC) but is not a sensitive indicator of an organism's reaction to stimulation under isoflurane anesthesia. Although nonsignificant, consistent changes occurred for stimulus type under all three doses. They increased on the order of 1.5-6 beats/minute, under the condition of painful stimulation, which is minuscule when compared with a typical base rate ( 270 beats/minute). In addition, the significant difference in HR during stimulation was limited to stimulus type. This difference may be attributable to differing reactions of the animals to the two stimuli (i.e., HR decreased in relation to baseline values under the auditory condition, whereas it increased under the tactile stimulus condition) and would support a conclusion that HR is not a sensitive measure for stimuli detection during anesthesia.

The consistent changes in HR over all three doses of isoflurane suggest that the animal is no less able to detect stimuli at one dose than at the other two. There are three explanations for these findings. The first is the possibility that the animal feels the tactile stimulus at all doses but is not able to move at the two higher doses. This was reflected in the results of the variable of movement. A second possible explanation is that the variable of change in HR is not a sensitive measure of stimuli detection. A third is that the animal does not perceive the stimulus differently on any particular dose. The first two seem to be logical possibilities, since, if the third were true, there does not seem to be a reasonable explanation of the results dealing with the variable of movement. If the animals did not feel anything at any dose, they should not have moved in response to tailpinch, but they did at 0.75 MAC. In addition, the analysis of ECoG variables, under condition of auditory stimulation, supports the notion that the animals are detecting stimuli to varying degrees at the different concentrations.

\section{Movement}

Movement is the typical measure used to determine whether animals experience stimulation during anesthesia (Dripps et al., 1977; Merkel \& Eger, 1963; Munson, 1970). Consistent with the literature, we found an inverse relationship between dose (MAC) and gross motor movement. However, in contrast with the literature, we did not find that greater than $50 \%$ of the rats exhibited movement at $0.75 \mathrm{MAC}$, which, by definition, they should. Since this is critical to MAC, possible reasons for this are addressed.

Since $1 \mathrm{MAC}$ is defined as the condition under which $50 \%$ of the animals tested fail to respond to a painful stimulus, we would expect that a $0.75 \mathrm{MAC}$ would have had greater than $50 \%$ movement upon painful stimulation. However, our results showed $42 \%$ moving at $0.75 \mathrm{MAC}$. This result could also have three possible explanations. One possibility was to look for a flaw in the calibration of the vaporizer; however, the vaporizer was calibrated on two occasions with a state-of-the-art mass-spectronieter, with consistent readings.

Another possibility was that the tailpinch was not painful. Care was taken to ensure consistency in the use of the clip, such that all animals had their tails pinched with the same amount of force. Five of 12 rats did move when pinched, indicating discomfort. In addition, bruising of the tail occurred in some instances, which would seem to indicate pressure of some intensity. Furthermore, none of the animals moved in response to the other, nonpainful, auditory stimulus.

The third possibility was that the percent of isoflurane reported to be $1 \mathrm{MAC}$ is in error. This seems unlikely, since there has not been a body of literature questioning the value of 1.38, as reported by Eger (1985) and White et al. (1974). In light of the evidence, no conclusive statement can be made as to the reason why the re- 
sults differed from what would be expected from the definition of MAC.

Our results are different from the standard table values for MAC (Eger, 1985; White et al., 1974). One of the reasons for this discrepancy may be the use of movement as the criterion for MAC values. The results in this experiment strongly suggest that movement is not the best predictor (i.e., it was the least sensitive measure, with ECoG the most sensitive measure of anesthetic depth). On the basis of previously reported MAC levels for isoflurane (Merkel \& Eger, 1963), we would have expected to observe at least $50 \%$ of the animals to move at $0.75 \mathrm{MAC}$, and they did not.

Taken together, these results support the notion that animals may be experiencing stimulation without being able to react via a motor response. Anesthetic depth may be partitioned in a modality-specific fashion. Furthermore, this study suggests that the ECoG acts as an early indicator of stimulus perception before more gross indicators (e.g., body movement) become apparent. These results lend further support to the caveat that measurement of the depth of anesthesia may depend heavily upon the modality we choose to measure (Gibbs, Larach, Skeehan, \& Schuler, 1989; Miller, 1990). It also suggests that the use of EEG in a human clinical setting would aid in detection of painful stimulus perception before such gross indicators as movement become apparent.

Finally, circadian effects were visually apparent in the records and were significant during baseline periods for HR, PP ECoG, and respiration. These reflect differences dependent upon time of day and were not emphasized, since the action of the anesthetic is of greatest concern. When determining the differences in action using changes in dependent measure, circadian variation was not profound, suggesting that there is no optimal time of day for isoflurane usage, unlike other popular inhalation anesthetics.

\section{REFERENCES}

Bahlman, S. H., Eger, E. I., \& Cromwell, T. H. (1972). Anesthetics amnesia. Anesthesiology, 36, 91 .

Barraco, R. A., Phillis, J. W., Campbell, W. R., Marcantonio, D. R., \& SALAH, R. S. (1989). The effects of central injections of adenosine analogs on blood pressure and heart rate in the rat. Neuropharmacology, 25, 675-680.

Bergman, B. M., Winter, J. B., Rosenberg, R. S., \& ReschtafFen, A. (1987). NREM sleep with low-voltage ECoG in the rat. Sleep, 10, 1-10.

Bolles, R. C. (1982). Endorphins and behavior. Annual Review of Physiology, 33, 87-101.

CHEEK, D. (1962). Importance of recognizing that surgical patients behave as though hypnotized. American Journal of Clinical Hypnosis, 4, 227-236.

CHEEK, D. (1964). Further evidence of persistence of hearing under chemoanesthesia: A detailed case report. American Journal of Clinical Hypnosis, 7, 55-59.

Clark, D. L., Hosick, E. C., Adam, N., Castro, A. D., Rosner, B. S., \& NeIGH, J. L. (1973). Neural effects of isoflurane (forane) in man. Anesthesiology, 39, 261-270.

Clark, D. L., \& Rosner, B. S. (1973). Neurophysiologic effects of general anesthetics: The electroencephalogram and sensory evoked responses in man. Anesthesiology, 38, 564-582.

Cromwell, T. H., Eger, E. L., Stevens, W. C., \& Dolan, W. M.
(1971). Forane uptake, excretion and blood solubility in man. Anesthesiology, 35, 401-408.

Davison, L. A., Steinhelber, J. C., Eger, E. L., \& Stevens, W. C. (1975). Psychological effects of halothane and isoflurane anesthesia. Anesthesiology, 43, 313-324.

DRIPPS, R. D., ECKENHOFF, J. E., \& VANDAM, L. D. (1977). Introduction to anesthesia: The principles of safe practice (5th ed.). Philadelphia: W. B. Saunders.

EGER, E. L. (1985). Isoflurane (forane): A compendium and reference (2nd ed.). Madison, WI: Anaquest.

Eger, E. L., Saidman, L. J., \& Brandstater, B. (1965). Minimum anesthetic concentration: A standard of anesthetic potency. Anesthe siology, 26, 756-763.

Eger, E. L., Stevens, W. C., \& Cromwell, T. H. (1971). The electroencephalogram in man: Anesthesia with forane. Anesthesiology, 35, 504-508.

EMLEN, S. T., \& KeM, W. (1963). Activity rhythm in peromyseus: Its influence on rates of recovery from Nembutal. Science, 142, 1682-1683.

Engelhardt, W., Carl, G., Dierks, T., \& Maurer, K. (1991). Electroencephalographic mapping during isoflurane anesthesia for treatment of mental depression. Journal of Clinical Monitoring, 7, 23-29.

FANSELOW, M. S. (1981). Naloxone and Pavlovian fear. Learning \& Mo tivation, 12, 398-419.

Gibbs, M. B., Larach, D. R., Skeehan, T. M., \& Schuler, H. G. (1989). Halothane induces depressor responses to noxious stimuli in the rat. Anesthesiology, 70, 503-510.

Gregory, G. A., Eger, E. I., \& Munson, E. S. (1969). The relationship between age and halothane requirement in man. Anesthesiology, 30, 488-491.

James, P. D., Volgyesi, G. A., \& Burrows, F. A. (1986). Alpha pattern ECoG during pediatric cardiac operations under isoflurane anesthesia. Anesthetic Analogies, 65, 525-528.

Loper, K., Reitan, J., Bennet, H., Benthuysen, J., \& Snook, L. (1987). Comparison of halothane and isoflurane for rapid anesthetic induction. Anesthetic Analogies, 66, 766-768.

MCGiNTY, D. J. (1967). Neural control of sleep patterns in the rat. Ann Arbor, MI: University Microfilms.

Meadows, P., Phillips, J. H., \& Davey, G. C. L. (1988). Tailpinch elicited eating in rats (Rattus norvegicus) and hamsters (Mesocricetus auratus). Physiology \& Behavior, 43, 429-433.

MERKEL, G., \& EGER, E. (1963). A comparative study of halothane and halopropane anesthesia including a method for determining equipotency. Anesthesiology, 24, 346-357.

Miller, R. D. (1990). Anesthesia (3rd ed.) New York: Churchill Livingston.

MunSon, E. S. (1970). Effects of hypothermia on anesthetic requirements in rats. Laboratory Animal Care, 20, 1109-1113.

Munson, E. S., Martucci, R. W., \& Smith, R. E. (1970). Circadian variations in anesthetic requirement and toxicity in rats. Anesthesiology, 32, 507-514.

NAIR, V., \& CASPER, R. (1969). The influence of light on daily rhythms in hepatic drug metabolizing enzymes in the rat. Life Sciences, $\mathbf{8}$, 1291-1298.

Nayak, A., Roy, R. J., \& Sharma, A. (1994). Time-frequency spectral representation of the EEG as an aid in the detection of depth of anesthesia. Annals of Biomedical Engineering, 22, 501-513.

PauCA, A. L., \& DripPs, R. D. (1973). Clinical experience with isoflurane (forane). Preliminary communication. British Journal of Anesthesia, 45, 697-703.

Pauley, J. E., \& Scheving, L. E. (1964). Daily fluctuation in biogenic amines in the rat brain. American Journal of Physiology, 214, 166-168.

PEARSON, R. (1961). Response to suggestions given under general anesthesia. American Journal Of Clinical Hypnosis, 4, 106-108.

Rampil, L. J., Weiskopf, R. B., Eger, E. L., Johnson, B. H., Holmes, M. A., \& Donegan, J. H. (1988). 1653 and isoflurane produce similar dose-related changes in the electroencephalogram of pigs. Anesthesiology, 69, 298-302.

Reddy, R. V., Moorthy, S. S., Mattice, T., Dierdorf, S. F., \& Deitch, R. D. (1992). An electroencephalographic comparison of effects of propofol and methohexital. Electroencephalography \& Clinical Neurophysiology, 83, 162-168. 
ReinberG, A., \& HalberG, F. (1971). Circadian chronopharmacology. Annual Review of Pharmacology \& Toxicology, 11, 455-492.

Roberts, P., Turnbull, M. J., \& Winterburn, A. (1970). Diurnal variation on sensitivity to and metabolism of barbiturates in the rat. $E u$ ropean Journal of Pharmacology, 12, 375-377.

SCHWILdEN, H., \& StOECKEL, H. (1987). Quantitative ECoG analysis during anesthesia with isoflurane in nitrous oxide at $1.3 \& 1.5 \mathrm{MAC}$. British Journal of Anesthesiology, 59, 738-745.

Shapiro, N. (Executive Producer) (1996, October 27). Dateline NBC. New York: National Broadcasting Company.

Stevens, W. C., Dolan, W. M., Gibbons, R. T., White, A., Eger, E. I., Miller, R. D., DE JoNG, R. H., \& ElashofF, R. M. (1975). Minimum alveolar concentrations (MAC) of isoflurane with and without nitrous oxide in patients of various ages. Anesthesiology, 42, 197-200.

Thomsen, C. E., Christensen, K. N., \& Rosenfalck, A. (1989). Computerized monitoring of depth of anesthesia with isoflurane. British Journal of Anesthesiology, 63, 518-519.

Tinker, J. H., Sharbrough, F. W., \& Michenfelder, J. D. (1977). Anterior shift of the dominant EEG rhythm during anesthesia in the Java monkey: Correlation with anesthetic potency. Anesthesiology, 46, 256-259.

White, P. F., JoHNSTON, R. R., \& EgER, E. I. (1974). Determination of anesthetic requirements in rats. Anesthesiology, 40, 52-57.
Yli-Hankala, A., Eskola, H., \& Kaukinen, S. (1989). ECoG spectral power during halothane anesthesia: A comparison of spectral bands in the monitoring of anesthesia level. Acta Anesthesia Scandinavica, 33, 304-308.

\section{NOTES}

1. Calibration was performed in conjunction with the Director of Audiology in the Department of Otolaryngology at Saint Louis University Health Sciences Center

2. Our attempt to maximally spread out the isoflurane concentrations was limited by the following considerations. Exceeding the lower limit would be unethical, since the animal would be subjected to painful stimuli while being more or less awake. The upper limit is set by both the absolute depth of anesthesia and its interaction with time. Isoflurane is a respiratory and blood pressure depressant. In addition, there is an interaction between depth of anesthesia and its duration, which are compounded. Hence, the above considerations and the length of time under the anesthetic required by the study demanded that we keep the doses studied in a fairly restricted range.

(Manuscript received November 4, 1996; revision accepted for publication January $21,1997$. ) 